\title{
Neoadjuvant chemotherapy for locally advanced cervical cancer
}

\author{
Takashi Iwata $^{1}$, Azumi Miyauchi ${ }^{1}$, Yukako Suga ${ }^{1}$, Hiroshi Nishio ${ }^{1}$, Masaru Nakamura ${ }^{1}$, Akiko Ohno ${ }^{1}$ \\ Nobumaru Hirao ${ }^{1}$, Tohru Morisada ${ }^{1}$, Kyoko Tanaka ${ }^{1}$, Hiroki Ueyama ${ }^{1}$, Hidemichi Watari ${ }^{2}$, Daisuke Aoki ${ }^{1}$ \\ ${ }^{1}$ Department of Obstetrics and Gynecology, Keio University School of Medicine, Tokyo, Japan; ${ }^{2}$ Department of Obstetrics and Gynecology, \\ Hokkaido University Graduate School of Medicine, Sapporo, Hokkaido, Japan \\ Correspondence to: Takashi Iwata, MD, PhD. Department of Obstetrics and Gynecology, Keio University School of Medicine, 35 Shinanomachi, \\ Shinjuku-ku, Tokyo, Japan. Email: iwatatakashi@1995.jukuin.keio.ac.jp.
}

\begin{abstract}
Neoadjuvant chemotherapy followed by surgery (NCS) has not been fully evaluated clinically. Currently, the main regimen of neoadjuvant chemotherapy (NAC) used in NCS includes cisplatin. The antitumor effects of NAC reduce lymph node metastasis and the tumor diameter in patients prior to surgery, and this can reduce the number of high risk patients who require postoperative radiation therapy. Many randomized controlled trials (RCTs) have examined the long-term prognosis of NCS compared to primary surgery, but the utility of NCS remains uncertain. The advent of concurrent chemoradiotherapy (CCRT) has markedly improved the outcome of radiotherapy (RT), and CCRT is now used as a standard method in many cases of advanced bulky cervical cancer. NCS gives a better treatment outcome than radiation therapy alone, but it is important to verify that NCS gives a similar or better outcome compared to CCRT.
\end{abstract}

Keywords: Neoadjuvant chemotherapy (NAC); cervical cancer

Submitted Jan 22, 2015. Accepted for publication Aug 08, 2015.

doi: $10.21147 /$ j.issn.1000-9604.2016.02.13

View this article at: http://dx.doi.org/10.21147/j.issn.1000-9604.2016.02.13

\section{Introduction}

Cervical cancer is the second most common cancer in females worldwide, with 500,000 new patients and 300,000 deaths due to this cancer reported globally each year (1). Among cervical cancer cases, $80 \%$ occur in developing countries (2) and about $70 \%$ are identified as advanced cancer (3). Treatment of cervical cancer is currently conducted based on the 2009 Federation of Gynecology and Obstetrics (FIGO) staging system (4), with FIGO stage IB1 and IIA1 cases treated by surgery or radiotherapy (RT) if no lymph node metastasis is observed. In contrast, the appropriate treatment for cervical cancer of stages IB2, IIA2, and IIB remains uncertain. Major treatment options include radical surgery with or without postoperative RT, neoadjuvant chemotherapy (NAC) followed by Radical surgery with or without postoperative RT, and concurrent chemoradiotherapy (CCRT). Radical surgery usually entails type III radical hysterectomy including pelvic lymphadenectomy with or without para-aortic lymphadenectomy (5). For stage IB2 and IIA2 cervical cancer, the National Comprehensive Cancer Network clinical guidelines mainly recommend CCRT (category 1) and to a lesser degree radical hysterectomy with pelvic lymphadenectomy and para-aortic lymph node sampling (category 2b). Stage IIB or more advanced cases are treated by RT because surgery is not applicable in these cases. However, the prognosis is poor and the five-year survival rate is about $40 \%$ (6).

Cervical cancers are sensitive to chemotherapy, and thus trials of chemotherapy combined with radiotherapy or surgery continue to be performed. In 1999, the National Cancer Institute recommended CCRT as the standard approach for locally advanced cervical cancer, based on a $30-50 \%$ decrease in mortality (7-10). In Europe, Japan and Latin countries, NAC followed by surgery (NCS) has emerged as a valid alternative and several pilot studies have shown a large benefit over RT alone in terms of overall survival (OS) and disease-free survival (DFS) (11). The best evaluation of the addition of chemotherapy to surgery is through a direct comparison with surgery alone, But when compared with primary surgery, the efficacy of NCS has 
not been determined in previous randomized controlled trials (RCTs) $(5,12-14)$ or observational studies (15-17). Recent reviews of NCS for cervical cancer have shown no significant benefit in OS over surgery alone $(18,19)$.

\section{Advantages and disadvantages of neoadjuvant chemotherapy (NAC) for cervical cancer}

The benefits of NCS are improvement of the radical cure rate, safety, a possible increase in the number of cases for which surgery is applicable due to the reduced tumor size, and suppression of remote metastasis by elimination of minute metastatic lesions. Zhang et al. (20) found that the rate of intrapelvic lymph node metastasis was significantly decreased in patients treated with NCS compared to those who underwent primary surgery because apoptosis in lymph nodes was induced by NAC. Further advantages of NCS include drugs reaching the tumor more easily because blood circulation to the tumor is not hampered by surgery or RT; and maintenance of hematopoiesis since there is no myelosuppression due to RT. In addition, NCS may be used as a substitute treatment in regions without facilities to conduct CCRT (21). However, NCS has disadvantages in that the treatment period is prolonged if chemotherapy is not successful, medical expenses are increased, patients may receive overmedication, and the tumor may progress before surgery. Autologous blood for autotransfusion also cannot be collected preoperatively in some cases and intraoperative blood transfusion may be needed due to anemia during chemotherapy. Therefore, the treatment approach should be selected after considering both the advantages and disadvantages of NCS.

\section{Clinical research on the efficacy of NAC}

NAC is more effective than RT alone based on a metaanalysis of RCTs (22), but comparison with surgery is required to clarify the efficacy of NCS. The following clinical studies performed a comparison of NCS with surgery alone (larify theRT) (Table 1).

(I) Sardi et al. (1997) (12)

A RCT was conducted for stage IB squamous cell carcinoma in NCS + RT and surgery + RT groups. The NAC regimen was cisplatin (CDDP) + vincristine (VCR) + bleomycin (BLM). There was no difference in the prognosis of patients with a tumor diameter $\leq 4 \mathrm{~cm}$ between the two groups, but the NCS group had a higher surgery completion rate in patients with a tumor diameter $>4 \mathrm{~cm}$.
These findings suggest that NCS can significantly prolong OS by reducing poor pathological prognostic factors.

(II) Napolitano et al. (2003) (13)

A RCT was conducted for IB-IIIB squamous cell cancers in NCS $( \pm$ RT) and surgery $( \pm$ RT) groups. The NAC regimen was CDDP + VCR + BLM. In the NCS group, extensive total hysterectomy was required in some cases. NAC contributed to prolonged survival in stage IB-IIB cases, but there was no significant difference in stage IIB cases.

(III) Cai et al. (2006) (14)

A RCT was conducted for stage IB squamous cell cancer and adenocarcinoma in NCS $( \pm \mathrm{RT})$ and surgery $( \pm \mathrm{RT})$ groups. The NAC regimen was CDDP + 5-fluorouracil (5-FU). Antitumor effects of NAC were observed in $84.2 \%$ of the patients (CCR: 7.7\%, PR: 76.9\%, SD: 15.4\%). The lymph node metastasis rate was much lower in the NCS group than in the surgery group $(9.6 \%$ vs. $29.6 \%, \mathrm{P}=0.04)$ and vascular space involvement was also significantly lower in the NCS group (9.6\% vs. $27.8 \%, \mathrm{P}=0.024)$. There was no significant difference in the incidence of parametrial invasion, but the five-year survival rate was significant higher in the NCS group compared to the surgery group (84.6\% vs. $75.9 \%, \mathrm{P}=0.0112$ ). These results suggest that NCS significantly prolongs long-term survival through suppression of lymph node metastasis and reduction of poor pathological prognostic factors.

(IV) Katsumata et al. (2006) (5)

A RCT was conducted for stage IB2-IIB squamous cell cancers in NCS $( \pm$ RT) and surgery ( \pm RT) groups. The $\mathrm{NAC}$ regimen was BLM + VCR + MitomycinC (MMC) + CDDP. The four-year survival rates were $74.6 \%$ in the NCS group and $74.4 \%$ in the surgery group, with no significant difference between the groups. It should be noted that termination of this trial was recommended during the trial since an interim analysis showed a low over all survival rate in patients receiving NAC.

(V) Eddy et al. (2007) (23)

A RCT (GOG141 study) was conducted for stage IB2 squamous cell cancer, adenosquamous carcinoma and adenocarcinoma in NCS $( \pm$ RT) and surgery ( \pm RT) groups. The NAC regimen was CDDP + VCR. There was no significant difference in recurrence and mortality rates, or in the total hysterectomy rate. Thus, this trial indicated no usefulness of NCS.

(VI) Chen et al. (2008) (24)

A RCT was conducted for stage IB2-IIB squamous cell cancer, adenosquamous carcinoma and adenocarcinoma 
Table 1 Demographic characteristics of 6 RCT studies

\begin{tabular}{|c|c|c|c|c|c|}
\hline Author & $\begin{array}{l}\text { FIGO } \\
\text { stage }\end{array}$ & Histology & $\begin{array}{c}\text { Comparison } \\
\text { (No. of patients) }\end{array}$ & Regimen of NAC & Cycles of NAC \\
\hline Cai (2006) (14) & IB & $\begin{array}{l}\text { SCC } \\
\text { AD }\end{array}$ & $\begin{array}{r}\text { NCS } \pm \text { RT [52] } \\
\text { Surgery } \pm \text { RT [54] }\end{array}$ & $\begin{array}{l}\text { CDDP } 75 \text { mg/m² day } 1 \\
\text { 5-FU } 24 \text { mg/kg day } 1-5\end{array}$ & Every 21 days for 2 cycles \\
\hline Eddy (2007) (23) & IB2 & $\begin{array}{c}S C C \\
A D, A S C\end{array}$ & $\begin{array}{r}\text { NCS } \pm \text { RT [145] } \\
\text { Surgery } \pm \text { RT [143] }\end{array}$ & $\begin{array}{l}\text { CDDP } 50 \mathrm{mg} / \mathrm{m}^{2} \text { day } 1 \\
\text { VCR } 1 \mathrm{mg} / \mathrm{m}^{2} \text { day } 1\end{array}$ & Every 10 days for 3 cycles \\
\hline Chen (2008) (24) & IB2-IIB & $\begin{array}{c}\text { SCC } \\
A D, A S C\end{array}$ & $\begin{array}{r}\text { NCS } \pm \text { RT [72] } \\
\text { Surgery } \pm \text { RT [70] }\end{array}$ & $\begin{array}{l}\text { CDDP } 100 \mathrm{mg} / \mathrm{m}^{2} \text { day } 1 \\
\text { MMC } 4 \mathrm{mg} / \mathrm{m}^{2} \text { day } 1-5 \\
5-\mathrm{FU} 24 \mathrm{mg} / \mathrm{kg} \text { day } 1-5\end{array}$ & Every 14 days for $2-3$ cycles \\
\hline
\end{tabular}

in NCS ( \pm RT) and surgery ( \pm RT) groups. The NAC regimen was $\mathrm{CDDP}+\mathrm{MMC}+5-\mathrm{FU}$. The success rate of NAC [complete response $(\mathrm{CR})+$ parcial response $(\mathrm{PR})]$ was $69.4 \%$, and in the NCS group PFS was significantly prolonged and the recurrence rate was significantly reduced in $\mathrm{CR}+\mathrm{PR}$ cases compared to stable disease (SD) + progression disease (PD) cases. The four-year survival rate was significantly greater in the NCS group compared to the surgery group (71.0\% vs. $58.0 \%, \mathrm{P}=0.041)$. However, in multivariate analysis, NAC was not an independent prognostic factor $(\mathrm{P}=0.074)$. The side effects of NAC were within the acceptable range, poor pathological prognostic factors were eliminated, and the prognosis was significantly improved in CR + PR cases with reduced tumor diameters.

\section{Review articles}

In the Cochrane review (18) conducted in 2010, 1,036 cases in six RCTs were analyzed, and the NCS group had better PFS compared to the surgery group. The local recurrence rate and remote metastasis rate were lower in the NCS group, but there was no significant difference and no improvement in OS. There was also no significant difference in the extended total hysterectomy rate. Thus, although NCS may improve the prognosis, these results did not prove greater efficacy of this method over primary surgery, and it was concluded that NSC should continue to be used only in clinical trials.

The Cochrane review was updated in 2012 (25) with addition of data for OS, local recurrence and remote 
metastasis in the RCT conducted by Chen et al. (24) This analysis showed significantly better PFS and OS in the NCS group compared to the surgery group, and the local recurrence rate was also significantly decreased in the NCS group. Chemotherapy is a systemic treatment, in contrast to localized treatment with RT, and therefore it was expected that NCS would decrease the remote metastasis rate. However, there was actually no significant difference in this rate between the NCS and surgery groups. Moreover, since postoperative RT was conducted together with NCS in many studies, it could not be concluded that the decreased local recurrence was due only to NCS. The total hysterectomy rate was evaluable based on the effect of NCS alone, but this rate showed no significant improvement with NCS. However, the lymph node metastasis rate and the incidence of parametrial invasion were lower in the NCS group compared to the surgery group.

\section{Markers reflecting the prognosis of NCS cases}

The benefits of NCS have yet to be proven and the method also has disadvantages of a prolonged treatment period, increased medical expenses, and the possibility of tumor progression. Therefore, selection of patients for whom NCS is likely to be successful and improve the prognosis is particularly important. In an analysis of serum metabolites after performance of NCS in 38 stage 1B2-IIB squamous cell cancer patients, Hou et al. found that L-valine and L-tryptophan were significantly higher in the pathological $\mathrm{CR}$ and PR groups compared to the SD group (26). The NAC regimen was PTX + CDDP.

Takatori et al. (27) investigated the effects of factors such as age, PS, clinical stage, histological type, tumor diameter, presence of lymph node metastasis before NCS, postoperative chemotherapy, squamous cell carcinoma antigen (SCC) levels before and after NCS, and success of anticancer drug therapy on prognosis after NCS. The regimen of NAC was irinotecan + CDDP. Among these factors, lymph node metastasis before treatment, SCC level after NCS, and the success rate of anticancer drug therapy were related to the prognosis. Thus, it was concluded that lymph node metastasis determined by CT is the most useful factor for selection of appropriate patients for NCS before the start of treatment.

Lu et al. (28) reviewed two RCTs and nine observational studies conducted from 1987 to 2012 to investigate whether the success rate of NCS is changed by histologic type. In patients in stage IIB and more advanced stages,
NCS significantly improved OS and PFS in squamous cell cancer compared to non-squamous cell cancers including adenocarcinoma.

\section{NCS for cervical cancer in pregnancy}

Cervical cancer in pregnancy accounts for about $2 \%$ of all cervical cancer cases and most of the cases are early-stage invasive cancers. Handling of continuation of pregnancy is unclear in these cases. If invasive cancer is found in the early stage, pregnancy may need to be terminated and total hysterectomy conducted. Alternatively, total hysterectomy can be delayed until the child is born, after the patient understands the risk of disease progression. In such cases, NCS can prevent progression of cancer and allow surgery to be delayed until the child is born. In an analysis of treatment outcomes in more than 47 pregnant females who underwent NSC, Zagouri et al. (29) found that the prognoses of mothers and babies were good. For NAC in pregnant women, drug administration after 14 weeks of pregnancy is recommended due to the reduced teratogenic risk after this period (30).

\section{NCS using drugs other than CDDP}

CDDP is currently the most important drug for cervical cancer and the regimen in many RCTs includes CDDP. However, clinical studies using drugs other than CDDP have also been reported. NCS has been performed using CDDP + PTX (31,32), but there has been no RCT of this treatment. The Japanese Gynecologic Oncology Group (JGOG) conducted a phase II multicenter clinical study on NCS in patients in stage Ib2-IVb (33). The success rate of NAC in CR + PR cases was $75.8 \%$ and the side effects were within an acceptable range. However, the success rate was not higher than that of NAC using CDDP + CPT-11 and there is currently no plan to conduct a phase III clinical trial.

\section{Conclusions}

The efficacy of NCS for cervical cancer has yet to be verified, but this treatment can reduce the risk factors of lymph node metastasis and vascular invasion and avoid the need for additional therapy such as postoperative RT. The prognosis may be improved by NCS in cases showing CR or those with elimination of lymph node metastasis by NAC. Thus, better treatment outcomes of NCS with a higher success 
rate are likely through combination with chemotherapy. NCS gives better outcomes than RT alone (22), but CCRT combined with CDDP also results in a better prognosis than RT alone and is currently the standard therapeutic approach for advanced bulky cervical cancer. Thus, a RCT comparing NCS and CCRT is required to investigate the utility of NCS.

\section{Acknowledgements}

None.

\section{Footnote}

Conflicts of Interest: The authors have no conflicts of interest to declare.

\section{References}

1. Jemal A, Bray F, Center MM, et al. Global cancer statistics. CA Cancer J Clin 2011;61:69-90.

2. Parkin DM, Bray F, Ferlay J, et al. Global cancer statistics, 2002. CA Cancer J Clin 2005;55:74-108.

3. Tewari KS, Sill MW, Long HJ 3rd, et al. Improved survival with bevacizumab in advanced cervical cancer. $\mathrm{N}$ Engl J Med 2014;370:734-43.

4. Pecorelli S, Zigliani L, Odicino F. Revised FIGO staging for carcinoma of the cervix. Int J Gynaecol Obstet 2009;105:107-8.

5. Katsumata N, Yoshikawa H, Kobayashi H, et al. Phase III randomised controlled trial of neoadjuvant chemotherapy plus radical surgery vs radical surgery alone for stages IB2, IIA2, and IIB cervical cancer: a Japan Clinical Oncology Group trial (JCOG 0102). Br J Cancer 2013;108:1957-63.

6. Zhao YB, Wang JH, Chen XX, et al. Values of three different preoperative regimens in comprehensive treatment for young patients with stage Ib2 cervical cancer. Asian Pac J Cancer Prev 2012;13:1487-9.

7. Keys HM, Bundy BN, Stehman FB, et al. Cisplatin, radiation, and adjuvant hysterectomy compared with radiation and adjuvant hysterectomy for bulky stage IB cervical carcinoma. N Engl J Med 1999;340:1154-61.

8. Morris M, Eifel PJ, Lu J, et al. Pelvic radiation with concurrent chemotherapy compared with pelvic and paraaortic radiation for high-risk cervical cancer. $\mathrm{N}$ Engl J Med 1999;340:1137-43.

9. Rose PG, Bundy BN, Watkins EB, et al. Concurrent cisplatin-based radiotherapy and chemotherapy for locally advanced cervical cancer. N Engl J Med 1999;340:1144-53.

10. Whitney CW, Sause W, Bundy BN, et al. Randomized comparison of fluorouracil plus cisplatin versus hydroxyurea as an adjunct to radiation therapy in stage IIB-IVA carcinoma of the cervix with negative paraaortic lymph nodes: a Gynecologic Oncology Group and Southwest Oncology Group study. J Clin Oncol 1999;17:1339-48.

11. Angioli R, Plotti F, Montera R, et al. Neoadjuvant chemotherapy plus radical surgery followed by chemotherapy in locally advanced cervical cancer. Gynecol Oncol 2012;127:290-6.

12. Sardi JE, Giaroli A, Sananes C, et al. Long-term followup of the first randomized trial using neoadjuvant chemotherapy in stage Ib squamous carcinoma of the cervix: the final results. Gynecol Oncol 1997;67:61-9.

13. Napolitano U, Imperato F, Mossa B, et al. The role of neoadjuvant chemotherapy for squamous cell cervical cancer (Ib-IIIb): a long-term randomized trial. Eur J Gynaecol Oncol 2003;24:51-9.

14. Cai HB, Chen HZ, Yin HH. Randomized study of preoperative chemotherapy versus primary surgery for stage IB cervical cancer. J Obstet Gynaecol Res 2006;32:315-23.

15. Kim HS, Kim JY, Park NH, et al. Matched-case comparison for the efficacy of neoadjuvant chemotherapy before surgery in FIGO stage IB1-IIA cervical cancer. Gynecol Oncol 2010;119:217-24.

16. Behtash N, Nazari Z, Ayatollahi H, et al. Neoadjuvant chemotherapy and radical surgery compared to radical surgery alone in bulky stage IB-IIA cervical cancer. Eur J Surg Oncol 2006;32:1226-30.

17. Cho YH, Kim DY, Kim JH, et al. Comparative study of neoadjuvant chemotherapy before radical hysterectomy and radical surgery alone in stage IB2-IIA bulky cervical cancer. J Gynecol Oncol 2009;20:22-7.

18. Rydzewska L, Tierney J, Vale CL, et al. Neoadjuvant chemotherapy plus surgery versus surgery for cervical cancer. Cochrane Database Syst Rev 2010;(1):CD007406.

19. Kim HS, Sardi JE, Katsumata N, et al. Efficacy of neoadjuvant chemotherapy in patients with FIGO stage IB1 to IIA cervical cancer: an international collaborative meta-analysis. Eur J Surg Oncol 2013;39:115-24.

20. Zhang H, Peng W, Zhang Y. Detection of cell apoptosis in pelvic lymph nodes of patients with cervical cancer after neoadjuvant chemotherapy. J Int Med Res 2014;42:641-50.

21. Basile S, Angioli R, Manci N, et al. Gynecological cancers in developing countries: the challenge of 
chemotherapy in low-resources setting. Int J Gynecol Cancer 2006;16:1491-7.

22. Neoadjuvant Chemotherapy for Locally Advanced Cervical Cancer Meta-analysis Collaboration.

Neoadjuvant chemotherapy for locally advanced cervical cancer: a systematic review and meta-analysis of individual patient data from 21 randomised trials. Eur J Cancer 2003;39:2470-86.

23. Eddy GL, Bundy BN, Creasman WT, et al. Treatment of ("bulky") stage IB cervical cancer with or without neoadjuvant vincristine and cisplatin prior to radical hysterectomy and pelvic/para-aortic lymphadenectomy: a phase III trial of the gynecologic oncology group. Gynecol Oncol 2007;106:362-9.

24. Chen H, Liang C, Zhang L, et al. Clinical efficacy of modified preoperative neoadjuvant chemotherapy in the treatment of locally advanced (stage IB2 to IIB) cervical cancer: randomized study. Gynecol Oncol 2008;110:308-15.

25. Rydzewska L, Tierney J, Vale CL, et al. Neoadjuvant chemotherapy plus surgery versus surgery for cervical cancer. Cochrane Database Syst Rev 2012;12:CD007406.

26. Hou Y, Yin M, Sun F, et al. A metabolomics approach for predicting the response to neoadjuvant chemotherapy in cervical cancer patients. Mol Biosyst 2014;10:2126-33.

27. Takatori E, Shoji T, Omi H, et al. Analysis of prognostic factors for patients with bulky squamous cell carcinoma of the uterine cervix who underwent neoadjuvant chemotherapy followed by radical hysterectomy. Int J Clin

Cite this article as: Iwata T, Miyauchi A, Suga Y, Nishio H, Nakamura M, Ohno A, Hirao N, Morisada T, Tanaka K, Ueyama H, Watari H, Aoki D. Neoadjuvant chemotherapy for locally advanced cervical cancer. Chin J Cancer Res 2016;28(2):235-240. doi: 10.21147/j.issn.1000-9604.2016.02.13
Oncol 2015;20:345-50.

28. He L, Wu L, Su G, et al. The efficacy of neoadjuvant chemotherapy in different histological types of cervical cancer. Gynecol Oncol 2014;134:419-25.

29. Zagouri F, Sergentanis TN, Chrysikos D, et al. Platinum derivatives during pregnancy in cervical cancer: a systematic review and meta-analysis. Obstet Gynecol 2013;121:337-43.

30. Amant F, Van Calsteren K, Halaska MJ, et al. Gynecologic cancers in pregnancy: guidelines of an international consensus meeting. Int J Gynecol Cancer 2009;19 Suppl 1:S1-12.

31. Mori T, Hosokawa K, Sawada M, et al. Neoadjuvant weekly carboplatin and paclitaxel followed by radical hysterectomy for locally advanced cervical cancer: longterm results. Int J Gynecol Cancer 2010;20:611-6.

32. Dueñas-Gonzalez A, López-Graniel C, González-Enciso A, et al. A phase II study of multimodality treatment for locally advanced cervical cancer: neoadjuvant carboplatin and paclitaxel followed by radical hysterectomy and adjuvant cisplatin chemoradiation. Ann Oncol 2003;14:1278-84.

33. Yamaguchi S, Nishimura R, Yaegashi N, et al. Phase II study of neoadjuvant chemotherapy with irinotecan hydrochloride and nedaplatin followed by radical hysterectomy for bulky stage Ib2 to IIb, cervical squamous cell carcinoma: Japanese Gynecologic Oncology Group study (JGOG 1065). Oncol Rep 2012;28:487-93. 